\title{
An Updated View of Giant Molecular Clouds, Gas Flows and Star Formation in M51 with PAWS
}

\author{
S. E. Meidt, E. Schinnerer, A. Hughes, D. Colombo (MPIA), J. Pety \\ (IRAM), S. Garcia-Burillo (OAN), A. Leroy (NRAO), C. L. Dobbs \\ (Univ. Exeter), K. F. Schuster, C. Kramer, G. Dumas (IRAM), and \\ T. Thompson (OSU)
}

\begin{abstract}
We present an overview of the latest results from the PdBI Arcsecond Whirlpool Survey (PAWS, PI: E. Schinnerer), which has mapped CO(1-0) emission in the nearby granddesign spiral galaxy M51 at 40pc resolution. Our data are sensitive to GMCs above $10^{5} \mathrm{M}_{\odot}$, allowing the construction of the largest GMC catalog to date - containing over 1500 objects using the CPROPS algorithm (Rosolowsky \& Leroy 2006). In the inner disk of M51, the properties of the CO emission show significant variation that can be linked to the dynamical environment in which the molecular gas is located. We find that dynamically distinct regions host clouds with different properties and exhibit different GMC mass spectra, as well as distinct patterns of star formation. To understand how this sensitivity to environment emerges, we consider the role of pressure on GMC stabilization (including shear and star formation feedback-driven turbulence). We suggest that, in the presence of significant external pressure, streaming motions driven by the spiral arm can act to reduce the surface pressure on clouds. The resulting stabilization impacts the global pattern of star formation and can account for the observed nonmonotonic radial dependence of the gas depletion time. Our findings have implications for the observed scatter in the standard GMC relations and extragalactic star formation laws.
\end{abstract}

Keywords. galaxies: spiral — ISM: structure - ISM: kinematics and dynamics

\section{Introduction}

Observations of molecular gas at high resolution in nearby spiral galaxies are critical to test the emerging picture of how galactic environment influences the properties and evolutionary pathways of a galaxy's GMC population, and its global patterns of star formation. In this contribution we study the interacting Whirlpool galaxy M51 ( $D=7.6$ $\mathrm{Mpc}$ ) in its $\mathrm{CO}(1-0)$ line emission. The unparalleled high resolution of the PAWS (PdBI Arcsecond Whirlpool Survey; Schinnerer et al., in prep; see Figure 1) data, combined with exceptional multi-wavelength coverage, makes it an ideal target for examining the influence of bar and spiral instabilities - and more generally, dynamical galactic environment on the organization of the ISM and global star formation patterns.

Our detailed analysis of the molecular gas in M51 - including its GMC population so far suggests that several mechanisms control its structure and evolution. Deviations in the gravitational potential from axisymmetry (i.e. the nuclear bar and two-armed spiral) induce shear, shocks, and streaming motions in the gas flow, leading to changes in the local gas surface density. The spiral arms, in particular, build up a high density reservoir and encourage agglomeration and cloud collision, which form the highest mass clouds (see Figure 1).

These changes in gas density subsequently alter the pattern of radiation from recent star formation, which occurs on the convex side of the spiral arm, and therefore also the 

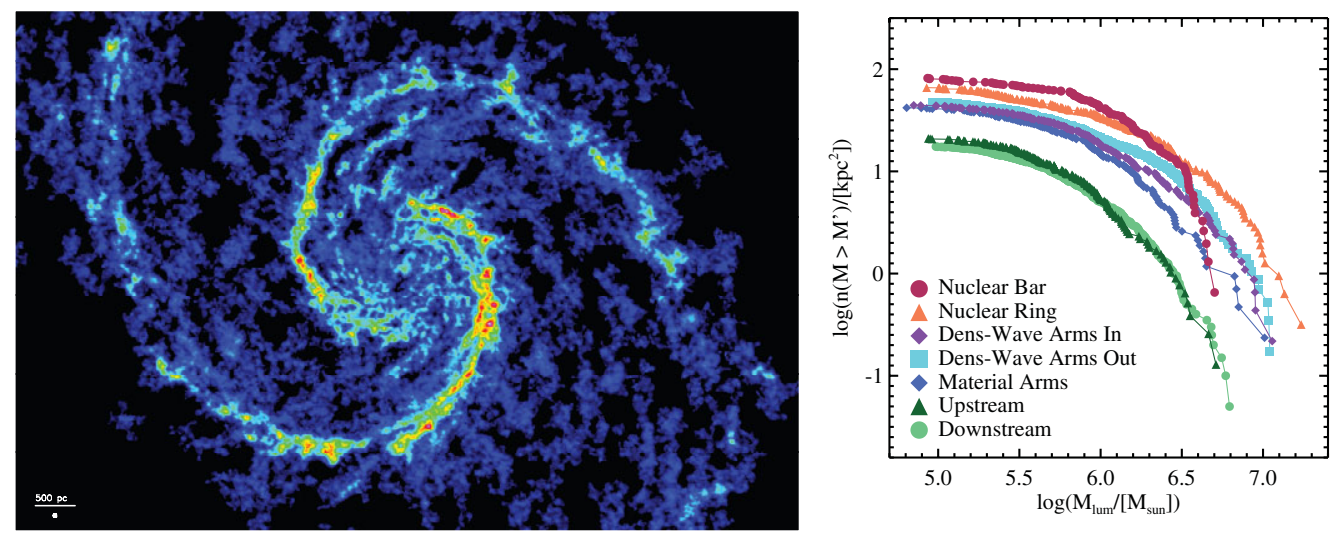

Figure 1. (Left) PAWS map of the integrated $\mathrm{CO}(1-0)$ line emission in the central $9 \mathrm{kpc}$ of M51, shown with square root intensity scaling. The bar in the bottom left denotes 500 pc 14 ". (Right) Cumulative mass spectra of catalogued GMCs in 7 different (dynamical) environments. All but the upstream and downstream interarm environments are defined with respect to zero-crossings in the torque profile in Figure 2, which mark the locations of changes in the direction of gas flow.

mechanical energy input from newly formed OB stars, stellar winds and supernovae. As clouds pass from the downstream side of one spiral arm to the upstream side of the next they are subject to stellar feedback as well as background shear. Shear induces cloud dispersal and acts to remove the record of cloud formation inherited in the spiral arm.

The galactic environment is thus very dynamic, and it is not clear under which conditions the developed static models apply (e.g. Krumholz et al. 2009). As described in the next section, the star formation properties of clouds appear highly sensitive to the kinematics and organization of the gas. To explore this link we have performed a detailed study of gas kinematics on the scales of individual GMCs. Depending on location in the disk, gas is driven radially in- or outward and is occasionally halted along its path (Meidt et al. 2012b, in prep.). Whether gas is in motion or stationary influences the build-up of the star-forming gas reservoir, the accumulation of mass in to clouds, and the eventual collapse to form stars.

\section{Radial Variation in the star formation efficiency}

Close inspection of young stellar tracers in relation to the CO morphology reveals that the pattern of M51's star formation shows a strong radial dependence (Schinnerer et al. 2012 , in prep.). In particular, there is a marked absence of strong star formation between $35 " \lesssim R \lesssim 60 "$ (as well as in zone of the nuclear bar $\mathrm{R}<25 "$ ) in either $\mathrm{H} \alpha$ or 24 $\mu m$ emission (Schinnerer et al. 2012, in prep). As a result, the measured star formation efficiency (SFE), and hence gas depletion time $\tau_{d e p}=\Sigma_{H_{2}} / \Sigma_{S F R}$ shows a non-monotonic radial dependence (Figure 2 and see also Leroy et al. 2009). Knapen et al. (1992) identified this same pattern and loosely interpreted this as a dependence on location relative to spiral arm resonances. This is demonstrated in Figure 2 comparing $\tau_{d e p}$ to the radial profile of present-day torques (Meidt et al. 2012, in prep), which switch from negative to positive at the location of corotation between the disk and the non-axisymmetric structure, driving radially inward and outward streaming motions, respectively. In Meidt et al. (2012, in prep) we argue that the inverse correlation of $\tau_{\text {dep }}$ with torques $\Gamma$ portrayed in Figure 2 reflects the sensitivity of clouds to their (non-circular) motion in the rotating 


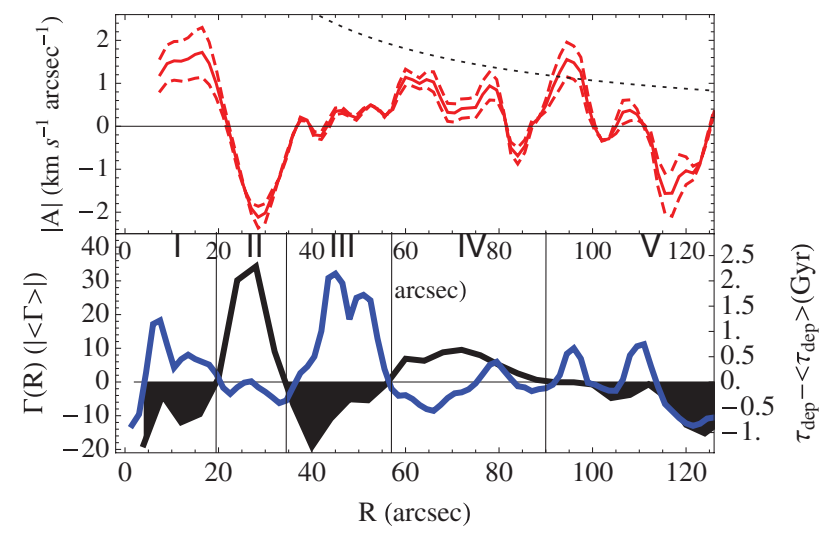

Figure 2. (Bottom) Azimuthally-averaged torques (black and white) and molecular gas depletion time (or inverse star formation efficiency; blue) calculated in 1.5" radial bins. Each crossing from negative to positive torque corresponds to the location of the corotation resonance (CR) of the structure: inside CR material is driven radially inward and outside material moves outward. (Top) Shear measured by the Oort constant A in the bar and spiral arm regions, calculated from azimuthal streaming velocities decomposed by Meidt et al. (2012, in prep.). Background shear due to differential rotation is shown in black dotted line. Shear in the spiral arm counters the background shear only between 18 " $\lesssim R \lesssim 28$ " and further out near $R \sim 85$ " and $R \sim 100$ ".

frame, the magnitude of which is shown in Figure 3. Below we describe this in terms of dynamical pressure, in consideration of other potential sources of cloud stability.

\section{Sources of cloud stability}

Shear As the locations of strong non-circular motions and enhanced surface density, spiral arms are preferentially sites of reduced shear (i.e. Kim \& Ostriker 2008). This also makes them favored sites of star formation since, in the absence of shear to counter selfgravity, gas can form coherent cloud structures that become gravitationally unstable. In M51, though, we find that the link between reduced shear and star formation does not hold uniformly along the spiral arms. The region of lowest shear in the spiral zone 35 " < $R<60$ " coincides with where we find a pronounced increase in $\tau_{d e p}$ (or decrease in SFE; see Figure 2). The low shear in this case should favor cloud collapse and star formation. This combination of low shear and low star formation is in contrast to the neighboring zone to larger galactocentric radius that shows a similar, if not higher, degree of shear but relatively more star formation. To prevent SF on cloud scales between 35 " $<R<60$ " another source of stability is clearly required.

Turbulence/SF feedback An enhancement in turbulent support in the zone 35 " $<R<$ $60 "$ compared to clouds from neighboring zones could potentially prevent clouds there from collapsing and forming stars. However, our high resolution PAWS observations, where the line-of-sight velocity profiles sample turbulent velocities above $5 \mathrm{~km} \mathrm{~s}^{-1}$ on cloud scales do not show such a pattern (Pety et al. 2012, in prep.; Colombo et al. 2012, in prep.). Moreover, the absence of star formation in this zone rules out cloud support from turbulence driven by stellar winds/feedback.

Dynamical Pressure The most compelling source of stability comes from dynamical pressure. Clouds embedded in a medium undergoing non-circular motion feel a reduced surface pressure $P_{\text {surf }}$, as qualitatively described by the compressible Bernoulli equation (Meidt et al. 2012, in prep). This can lead to stabilization when clouds are dynamically coupled to their environment, i.e. when the internal and external pressures are similar, as measured to be the case in M51 (as well as in two lower mass systems, M33 and the 

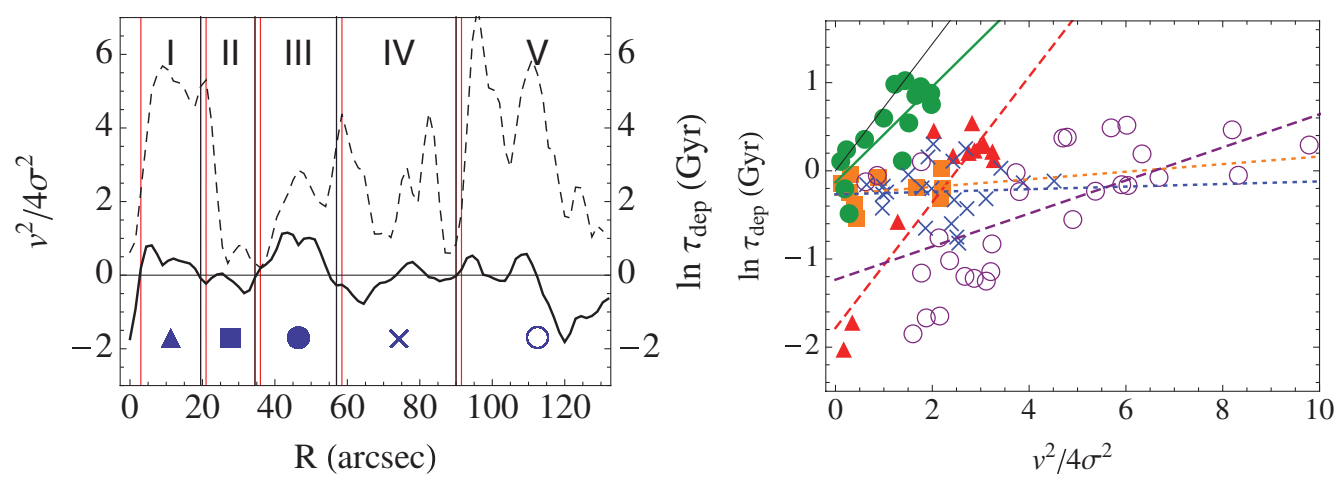

Figure 3. (Left) Profiles of $v_{S}^{2} / 4 \sigma^{2}$ (dashed black) and $\ln \tau_{\text {dep }}$ (solid black) throughout M51. Errors have been omitted for clarity. Vertical lines delineate five dynamical environments (defined in fig. 2; see Meidt et al. 2012, in prep.): (in order) nuclear bar, molecular ring, density wave spiral inside and outside CR, material spiral pattern. (Right) Scatter plot of $\ln \tau_{d e p}$ vs. $v_{S}^{2} / 4 \sigma^{2}$ extracted in 1.5" bins from the curves at right. Symbols are as indicated at left. Points fall along environment-unique lines with slopes $-(1+\gamma)$ and intercepts $\ln \tau_{d e p, 0}$, according to eq. 3.1. On average, the index $\gamma=-1.37 \pm 0.34$ measured from the best-fit slopes is consistent with (if slightly lower than) the indices measured in the same set of environments by Hughes et al. (2012) via direct fitting of the cloud mass spectra $(\langle\gamma\rangle=-1.72 \pm 0.39$ on average).

LMC, among others; Hughes et al. 2012). The changes to cloud boundedness as a result of dynamical pressure naturally lead to changes in the efficiency of star formation and hence gas depletion time $\tau_{d e p}=\Sigma_{g a s} / \Sigma_{S F R}$. Assuming that the process (or processes) responsible for the build-up of GMCs results in a mass spectrum $\mathrm{N}(>\mathrm{M}) \propto \mathrm{M}^{\gamma+1}$ we find

$$
\ln \frac{\tau_{d e p}}{\tau_{d e p, 0}} \approx-(1+\gamma) \frac{v_{S}^{2}}{4 \sigma^{2}} .
$$

where $\gamma$ is the cloud mass spectrum power-law index, $v_{S}^{2}$ is the magnitude of streaming motions (Meidt et al. 2012, in prep), $\sigma \sim 10 \mathrm{~km} \mathrm{~s}^{-1}$ is the observed velocity dispersion corrected for unresolved bulk motions and $\tau_{d e p, 0}$ is the 'fiducial' depletion time in the absence of streaming. As shown in the left panel of Figure 3, the radial variation in the $\tau_{d e p}$ echoes the radial dependence in the kinematic term $v_{S}^{2} / \sigma^{2}$, and the two appear similar to within an environment-dependent, radially varying scale factor $-(\gamma+1)$.

According to eq. (3.1) we can expect that streaming motions contribute to the scatter in the spatially-resolved Kennicutt-Schmidt law. Likewise, we expect longer depletion times where streaming motions are higher, and thus a decrease in $\tau_{d e p}$ from early to late-type spirals.

\section{References}

Kim, W.-T. \& Ostriker, E. C. 2002, ApJ, 570, 132

Knapen, J. \& Beckman, J. 1992, ApJ

Krumholz, M., \& McKee, C., F., Tumlinson, J. 2009, ApJ, 699, 850

Leroy, A. K., Walter, F., Bigiel, F., Usero, A., Weiss, A., Brinks, E., de Blok, W. J. G., Kennicutt,

R. C., Schuster, K. -F., Kramer, C., Wiesemeyer, H. W., \& Roussel, H. 2009, AJ, 1374670

Rosolowsky, E. \& Leroy, A. 2006,PASP, 118, 590 